\title{
SIRT3 deficiency is resistant to erastin-induced autophagy-dependent ferroptosis by inhibiting the AMPK/mTOR pathway and promoting glutathione peroxidase 4 levels
}

\section{Dandan Han}

China Agricultural University

Lili Jiang

China Agricultural University

Xiaolong Gu

Yunnan Agricultural University

Shimeng Huang

China Agricultural University

Jiaman Pang

China Agricultural University

Yujun Wu

China Agricultural University

Cuiping Feng

China-Japan Friendship Hospital

Jingdong Yin

China Agricultural University

Junjun Wang ( $\square$ wangjj@cau.edu.cn )

China Agricultural University https://orcid.org/0000-0001-9427-3824

Research

Keywords: Autophagy, Ferroptosis, SIRT3, AMPK-mTOR pathway, GPX4

Posted Date: January 7th, 2020

DOI: https://doi.org/10.21203/rs.2.20204/v1

License: (1) This work is licensed under a Creative Commons Attribution 4.0 International License.

Read Full License 


\section{Abstract}

Background: Ferroptosis, an autophagy-dependent cell death, is characterized by lipid peroxidation and iron accumulation, closely associated with pathogenesis of gestational diabetes mellitus (GDM). Sirtuin 3 (SIRT3) has positive regulation on phosphorylation of AMPK, related to maintainance of cellular redox homeostasis. However, whether SIRT3 can confer autophagy by activating the AMPK-mTOR pathway and consequently promote induction of ferroptosis is unknown.

Methods: We used human trophoblastic cell line HTR-8/SVneo and porcine trophoblastic cell line pTr2 to deterimine the mechanism of SIRT3 on autophagy and ferroptosis.

Results: The expression of SIRT3 protein was significantly elevated in trophoblastic cells exposed to high concentrations of glucose and ferroptosis-inducing compounds. Increased SIRT3 expression contributed to classical ferroptotic events and autophagy activation, whereas SIRT3 silencing led to resistance against both ferroptosis and autophagy. In addition, autophagy inhibition impaired SIRT3-enhanced ferroptosis. On the contrary, autophagy induction had a synergistic effect with SIRT3. Based on mechanistic investigations, SIRT3 depletion inhibited activation of the AMPK-mTOR pathway and enhanced glutathione peroxidase 4 (GPX4) level, thereby suppressing autophagy and ferroptosis. Furthermore, depletion of AMPK blocked induction of ferroptosis in trophoblasts.

Conclusions: We concluded that upregulated SIRT3 enhanced autophagy activation by promoting AMPKmTOR pathway and decreasing GPX4 level to induce ferroptosis in trophoblastic cells. SIRT3 deficiency was resistant to high glucose- and erastin-induced autophagy-dependent ferroptosis and is therefore a potential therapeutic approach for treating GDM.

\section{Background}

Gestational diabetes mellitus (GDM), one of the most common metabolic complications of pregnancy, is characterized by aberrant placental functions, resulting in a significantly increased risk of developing type 2 diabetes mellitus (T2DM) in both mother and child [1-3]. There are indications that induction of antiangiogenic factors and a proinflammatory milieu, including impaired trophoblast migration and invasion, are closely associated with abnormal placental function in development of GDM [4, 5]. Moreover, appropriate proliferation, migration and differentiation of trophoblasts dominate progression of steroids, hormones and cytokines secretion, vascularizationa and the immunological interface required for normal pregnancy [6-8]. Multiple studies verified that diabetic placenta was usually accompanied by abnormal trophoblast proliferation, autophagy and cell cycle control; thereby, development and progression of GDM was dramatically induced $[4,9,10]$. In addition, overproduction of reactive oxygen species (ROS) occurs in trophoblastic cells after stimulation with high glucose concentrations and superabundant accumulation of ROS can result; eventually, this continuous oxidative stress initiates induction and pathogenesis of GDM $[11,12]$. Elucidating preventive mechanisms against oxidative stress-triggered lipid 
metabolism disorder is expected to facilitate development of evidence-based treatment strategies for GDM.

Autophagy is programmed cell death, with an important role in a degradative pathway for eliminating excessive misfolded protein and damaged organelle aggregates [13]. Accumulation of ROS promotes autophagy-dependent cell death of trophoblasts by increasing lipid peroxidation and reducing activities of antioxidant enzymes [14, 15]. As a homeostatic dysfunction of autophagy is believed to be a cause of GDM, it is critical to explore how trophoblastic autophagy is physiologically regulated. Ferroptosis is a regulated cell death-exhibiting production of ROS, from iron-dependent formation of lipid peroxidation, rupture of mitochondrial outer membrane and collapse of cellular redox homeostasis $[16,17]$. However, crosstalk between autophagy and ferroptosis, as well as underlying mechanisms responsible for these two types of cell death, have not been fully elucidated.

Sirtuin 3 (SIRT3), a prototypical NAD ${ }^{+}$-dependent mitochondrial protein deacetylase, is responsible for bulk mitochondrial protein deacetylation that regulates metabolism and oxidative stress [18]. SIRT3 is involved in maintenance of mitochondrial function, including ATP generation, nutrient oxidation, ROS production, and even cell death $[18,19]$ A novel role of SIRT3 in regulating phosphorylation of activated protein kinase (AMPK) to promote autophagy was recently reported [20]. However, plausible crosstalk between AMPK and SIRT3 during oxidative stress-mediated cell death has apparently not been studied. Based on capability of AMPK to promote autophagy via mTOR and SIRT3 to inhibit oxidative stressinduced ferroptosis [20,21], we speculated that protective effects of SIRT3 on autophagy and ferroptosis would be associated with AMPK. Therefore, the objective was to investigate underlying mechanisms in SIRT3-mediated protection of trophoblasts against autophagy and ferroptosis, with a special focus on the AMPK-mTOR pathway.

\section{Materials And Methods}

\section{Reagents and antibodies}

DME/F12 medium, Opti-MEM medium and fetal bovine serum (FBS) were obtained from Gibco (Grand island, NY, USA). Dimethyl sulfoxide (DMSO, cat \#D2650), buthionine sulfoximine (BSO, cat \#19176), compound C (cat \#171260) and Z-VAD-FMK (cat \#V116) were purchased from Sigma-Aldrich Chemical (Sigma, St. Louis, MO, USA). Erastin (cat \#S7242), sorafenib (cat \#S7397), ferrostatin-1 (cat \#S7243), liproxstatin-1 (cat \#S7699), necrosulfonamide (cat \#S8251), RSL3 (cat \#S8155), rapamycin (Rapa, cat \#S1039), chloroquine (CQ, cat \#S4157) and bafilomycin A1 (Baf-A1, cat \#S1413) were acquired from Selleck Chemicals (Shanghai, China). Primary antibody to SIRT3 (cat \#10099-1-AP, 1:1000) was obtained from Proteintech Group (Chicago, IL, USA). Antibody for beclin1 (cat \#NB110-87318, 1:10000) was purchased from NOVUS (CA, USA). Primary antibody to LC3B (cat \#ab229327, 1:1000) and GPX4 (cat \#ab125066, 1:2000) was purchased from Abcam (Abcam, Cambridge, MA, USA). Antibodies against $\beta$ actin (cat \#4970, 1:1000), AMPK (cat \#4178, 1:1000), p-mTOR (cat \#5536, 1:1000), mTOR (cat \#2983, 1:1000) and anti-rabbit IgG (cat \#7074, 1:1500) were obtained from Cell Signaling Technology (Danvers, 
MA, USA). Anti-phospho-AMPKa1/2 (Thr183/172) polyclonal antibody (cat \#YP0575, 1:2000) was acquired from ImmunoWay (Plano, TX, USA).

\section{Cell culture and treatment}

Human trophoblastic cell line HTR-8/SVneo was acquired from BeNa culture collection (Beijing, China). Porcine trophectoderm ( $\mathrm{pTr}$ ) cell line was kindly provided by Prof. Zhenlong Wu (China Agricultural University, Beijing, China). These two cell lines were cultured in DME/F12 medium supplemented with $10 \%\left(\mathrm{v} / \mathrm{v}\right.$ ) heat-inactivated FBS at $37^{\circ} \mathrm{C}, 95 \%$ humidity, and $5 \% \mathrm{CO}_{2}$. For drug stimulation, cells were treated with erastin, sorafenib and BSO, with or without ZVAD-FMK, necrosulfonamide, and ferrostatin-1 for $24 \mathrm{~h}$ in the presence of high glucose concentrations, as indicated. For RNA interfence, cells were transfected with SIRT3 siRNA. Subsequently, the indicated SIRT3-silenced trophoblastic cells were exposed to erastin with or without ZVAD-FMK, necrosulfonamide, ferrostatin-1, rapamycin, chloroquine and compound $\mathrm{C}$ for $24 \mathrm{~h}$, in the presence of high glucose concentrations.

\section{Gene knockdown of SIRT3 by siRNA and transfection of cells}

Specific small interfering RNA (siRNA) duplexes against human and porcine SIRT3 gene were synthesized and transfected into human and porcine trophoblastic cells, respectively. Cells were incubated with the siRNA directed against SIRT3 using Lipofectamine 2000 reagent (Invitrogen, Carlsbad, CA, USA), according to manufacturer's instructions. After transection, cells were harvested and subjected to Western blot assay.

\section{Cell viability assay}

HTR-8/SVneo and pTr-2 cells were cultured in 96-well slides. After treatments, $100 \mu \mathrm{L}$ fresh medium containing 10\% Cell Counting Kit-8 (CCK-8) solutions (cat \#CK04; Dojingdo Laboratories, Kumamoto, Japan) was added and cells were incubated for $2 \mathrm{~h}$ at $37^{\circ} \mathrm{C}$. Absorbance was recorded at $450 \mathrm{~nm}$ using a microplate reader (Bio-Rad, Hercules, CA, USA). Cell viability (\%) was calculated using control cells as positive control ( $100 \%$ cell viability), whereas cell culture medium were used as blank control $(0 \%$ cell viability), according to the following:

\section{Iron assay}

Intracellular iron concentration was measured with an Iron Assay kit (cat \#ab83366; Abcam, Cambridge, MA, USA). Briefly, cells were lysed on ice, and then supernatants were collected after centrifugation at $13000 \times \mathrm{g}$ for $10 \mathrm{~min}$. Supernatants were co-incubated with $5 \mu \mathrm{L}$ Iron Reducer solution at $37^{\circ} \mathrm{C}$ for $30 \mathrm{~min}$. Subsequently, $100 \mu \mathrm{L}$ Iron Probe were added and incubated for $1 \mathrm{~h}$ at $37^{\circ} \mathrm{C}$ in dark; thereafter, absorbance was detected at $593 \mathrm{~nm}$ using a microplate reader.

\section{Glutathione (GSH) assay}


Relative GSH concentration in cell lysates was assessed using a Total Glutathione Quantification Kit (cat \#T419; Dojingdo Laboratories, Kumamoto, Japan). Cells were lysed using 5\% 5-sulfosalicylic acid solution. After centrifugation at $8,000 \mathrm{xg}$ for $10 \mathrm{~min}$, supernatants were collected and incubated with the substrate $5,5^{\prime}$-dithiobis (2-nitrobenzoic acid) at $37^{\circ} \mathrm{C}$ for $10 \mathrm{~min}$, which produced yellow 5-thio-2nitrobenzoic acid. Subsequently, $20 \mu \mathrm{L}$ substrate working solution was added and incubated for $10 \mathrm{~min}$ at room temperature. Absorbance was measured with a microplate reader at $412 \mathrm{~nm}$.

\section{Malondialdehyde (MDA) assay}

Analysis of lipid peroxidation was performed by quantification of MDA with a specific colorimatria kit (cat \#ab118970; Abcam, Cambridge, MA, USA) following manufacturer's instructions. Absorbance was measured with a microplate reader at $532 \mathrm{~nm}$, with MDA concentration calculated based on a standard curve.

\section{Reactive oxygen species (ROS) quantification}

Cells were incubated with $5 \mu \mathrm{M} 2^{\prime}, 7^{\prime}$-dichlorofluorescein diacetate (DCFH-DA) probe for $30 \mathrm{~min}$ at $37^{\circ} \mathrm{C}$. Fluorescence assays were evaluated and quantified using a fluorescence microplate reader (Tecan, Sunrise, Männedorf, Switzerland) at excitation/emission of 525/610 nm.

\section{Western blot analyses}

HTR8/SVeno and pTr2 cells were lysed using Total Protein Extraction Kit (cat \#KGP2100; KeyGEN, Nanjing, China). Protein concentration was quantified using a BCA Protein Assay kit (cat 323227; Thermo Fisher Scientific, Waltham, MA, USA). Equal amounts of protein were subjected to sodium dodecyl sulphate polyacrylamide gel electrophoresis (SDS-PAGE) prior to electro-transfer to a poly-vinylidine difluoride membrane (PVDF, cat \#ISEQ00010; Millipore, MA, USA). After blocking with 5\% skimmed milk, membranes were probed overnight at $4^{\circ} \mathrm{C}$ with specific primary antibodies, prior to incubation with corresponding secondary antibodies and protein was detected with an enhanced chemiluminescence method (ECL, Solarbio, Beijing, China).

\section{Statistical analyses}

Results were expressed as mean \pm standard deviation (SD). Statistical significance was assessed by Student's t-test or one-way analysis of variance (ANOVA), followed by post hoc Tukey's test. For all statistical analyses, $\mathrm{P}<0.05$ was considered significant.

\section{Results}

\section{SIRT3 is increased during trophoblast ferroptosis}

High glucose-induced trophoblast cell death contributes to pathogenesis of GDM [12]. High glucose concentrations induced ferroptotic events, including significant growth inhibition, intracellular iron accumulation, GSH depletion and lipid peroxidation in both human and porcine trophoblast cells 
(Supporting Fig. S1). To investigate whether erastin (a classical inducer of ferroptosis), sorafenib, buthionine sulfoximine (BSO, a GSH synthase inhibitor) could induce trophoblast ferroptosis, HTR8/SVeno and pTr2 cells were treated with erastin, sorafenib and BSO in high glucose condition, respectively. Erastin-, sorafenib- and BSO-mediated cell viability inhibition (Fig. 1a), redox-active iron accumulation (Fig. 1b), GSH depletion (Fig. 1c) and MDA production (Fig. 1d) were partly blocked by ferrostain-1 (a potent ferroptosis inhibitor), but not by necrosulfonamide (necroptosis inhibitor) nor ZVAD-FMK (apoptosis inhibitor).

As SIRT3 has critical roles in regulating oxidative stress and mitochondrial protein acetylation [18], we explored effects of various ferroptosis-inducing compounds on SIRT3 protein expression to determine whether SIRT3 has a pivotal regulatory role during ferroptosis. Cultivated trophoblasts exposed to erastin, sorafenib and BSO in the presence of high glucose had upregulation of SIRT3 protein in both human (HTR8/SVeno) and porcine (pTr2) trophoblast cells (Fig. 1e).

\section{Increased SIRT3 expression contributes to trophoblast ferroptosis}

To determine if increased SIRT3 is associated with induction of ferroptosis, siRNA of SIRT3 was introduced to HTR8/SVeno and pTr2 cells stimulated with erastin in high glucose condition. SIRT3 siRNA significantly decreased SIRT3 protein level in both HTR8/SVeno and pTr2 cells (Supporting Fig. S2). As expected, SIRT3 knockdown abolished high glucose and erastin-induced growth inhibition (Fig. 2a), redox-active iron overload (Fig. 2b), GSH depletion (Fig. 2c), MDA production (Fig. 2d) and intracellular ROS generation (Fig. 2e) in trophoblasts. Furthermore, ferrostatin-1 and liproxstatin-1 (two ferroptosis inhibitors) significantly enhanced survival rates of HTR8/SVeno cells after exposure to erastin in presence of high glucose (Supporting Fig. S3). Furthermore, erastin-mediated growth inhibition was markedly restored by ferrostatin-1 and liproxstatin-1 in SIRT3 depletion trophoblastic cells. However, ZVAD-FMK or necrosulfonamide failed to influence cell viability under these circumstances (Fig. 2f).

\section{Increased SIRT3 expression promotes autophagy activation}

To test whether high glucose exerts any influence on autophagy in cultivated trophoblastic cells, Western blot analysis was employed to determine conversion of LC3B-I to LC3B-II and protein level of beclin1. When trophoblastic cells were exposed to high glucose alone, there were significant increases in LC3B-II accumulation and beclin1 protein; furthermore, chloroquine (a lysosomal inhibitor that prevents autophagic degradation in lysosomes) and bafilomycin (a lysosomal inhibitor that prevents autophagosomes from fusion with lysosomes to form autophagosomes) dramatically elevated this increase. Conversely, rapamycin promoted high glucose-induced autophagy activation, evidenced by enhanced LC3B-II conversion and increased beclin1 protein expression (Supporting Fig. S4). To further clarify influence of autophagy activation on ferroptosis, markers of autophagy were examined by western blot analysis in both control siRNA and SIRT3 siRNA-treated cells. As expected, SIRT3 sliencing 
significantly blocked erastin-induced accumulation of LC3B-II (Fig. 3a) and remarkably decreased expression of beclin1 (Fig. 3b).

\section{Enhanced ferroptosis by SIRT3 is associated with autophagy activation}

Several lines of evidence reinforced the notion that ferroptosis is an autophagic cell death process. ${ }^{22-25}$ To evaluate whether autophagy is involved in SIRT3-mediated ferroptosis, rapamycin and chloroquine were exploited to activate or inhibit autophagy, respectively. There were superimposed effects on erastininduced growth inhibition (Fig. 4a), redox-active iron overload (Fig. 4b), GSH depletion (Fig. 4c), and MDA production (Fig. 4d) in trophoblasts stably transfected with SIRT3 siRNA followed by rapamycin exposure, compared to cells treated with SIRT3 siRNA alone. Conversely, chloroquine blunted ferroptotic events induced by erastin, evidenced by decreased growth inhibition (Fig. 4a), iron accumulation (Fig. 4b), GSH depletion (Fig. 4c), and lipid peroxidation (Fig. 4d).

\section{SIRT3 upregulates autophagy by promoting AMPK}

AMPK is a positive regulator of autophagy via suppressing mammalian target of rapamycin C1 (mTORC1) [26]. To study the role of AMPK in SIRT3-mediated autophagy activation, we initially detected AMPK activation after SIRT3 depletion using western blot. SIRT3 knockdown was associated with suppressed AMPK activation, evidenced by decreased protein phosphorylation of AMPK (Fig. 5a), as well as increased mTORC1 activation (Fig. 5b). The AMPK inhibitor, compound C, was used to further determine if AMPK is mechanistically implicated in effect of SIRT3 on autophagy. Protein expression of p-AMPK was decreased to $<30 \%$ in compound C-treated cells (Supporting Fig. S5). Furthermore, compound C abolished LC3-II conversion (Fig. 5c) and decreased expression of beclin1 (Fig. 5d) resulting from SIRT3 activation.

\section{Suppression of AMPK impairs SIRT3-enhanced ferroptpsis}

To explore whether AMPK activation triggers SIRT3-enhanced ferroptosis, compound $\mathrm{C}$ was used to inhibit phosphorylation of AMPK. Compound $\mathrm{C}$ not only inhibited phosphorylation of AMPK, it also reduced erastin-induced growth inhibition in human trophoblastic HTR8/SVeno cells (Fig. 6a). Similarly, pretreatment with compound $\mathrm{C}$ significantly abolished high glucose and erastin-induced redox-active iron accumulation (Fig. 6b), GSH depletion (Fig. 6c), and MDA production (Fig. 6d), in the absence or presence of SIRT3 SiRNA.

\section{Depletion of SIRT3 inhibits ferroptosis by increasing GPX4 level}

To assess the involvement of GPX4 in SIRT3-enhanced ferroptosis, cells were treated with RSL3, a GPX4 inhibitor. The results demonstrated that RSL3 significantly enhanced high glucose and erastin-induced 
growth inhibition (Fig. 7a), redox-active iron accumulation (Fig. 7b), GSH depletion (Fig. 7c), and MDA production (Fig. 7d), in the absence or presence of SIRT3 siRNA of human trophoblastic HTR8/SVeno cells. Compared to cells treated with control siRNA, trophoblasts stably transfection with SIRT3 siRNA rescued RSL3-induced growth inhibition (Fig. 7a), redox-active iron overload (Fig. 7b), GSH depletion (Fig. 7c), and MDA production (Fig. 7d).

\section{Discussion}

Oxidative stress disrupts placental tissue homeostasis and lipid metabolism $[13,15]$. There is increasing evidence iron-related oxidative stress may contribute to lipid oxidation and consequently produce distinct types of cell death, e.g. apoptosis, necrosis, ferroptosis and autophagy [16, 27, 28]. Mounting evidence has confirmed that ferroptosis, an iron- and lipotoxicity-dependent regulated cell death, is driven by an iron-catalyzed lipid peroxidation accumulation initiated via a Fenton reactions-triggered production of hydroxyl radicals and enzymatic lipoxygenase mechanisms [22, 29]. Lipophagy, intracellular lipid droplets degradation by autophagy, promotes ferroptotic cell death through induction of lipid peroxidation in hepatocytes, both in vivo and in vitro [30]. However, potential mechanistic consequences of how uncontrolled lipid peroxidation involving in autophagy-dependent ferroptosis are still under debate. In the present study, exposure to high glucose dramatically reduced cell viability and increased production of oxidants in trophoblasts, resulting in ferroptosis in both human and porcine trophoblast cells. We also reported that erastin, sorafenib and BSO can trigger accumulation of ferroptotic trophoblast in vitro.

Activation of SIRT3 is a major mechanism accounting for many lipid metabolic restriction-derived benefits to control mitochondrial function and mROS homeostasis [19, 31-33]. Overexpression of SIRT3 modulated protein acetylation and bolstered cellular resistance to mitochondrial stress and ferroptosis $[21,31,34]$. Therefore, effects and underlying mechanisms of SIRT3 on trophoblastic ferroptosis were explored in the current study. SIRT3 protein level was remarkedly upregulated after cultivated trophoblasts were exposed to ferroptosis-inducing agents in high glucose condition. Moreover, depletion of SIRT3 blocked oxidative stress and ferroptotic effects of high glucose and erastin. Therefore, we inferred that SIRT3 was involved in ferroptosis of trophoblastic cells.

Interestingly, there is a complex crosstalk between ferroptosis and autophagy through complex feedback loops $[23,35]$. Ferroptosis induction is coupled to an increase in turnover of MAP1LC3 and autophagosome formation, consistent with the notion that lipid peroxidation as well as oxidized lipids can promote autophagy activation $[16,36]$. Recent study indicates that clockophagy, a novel mode of selective autophagic degradation, facilitates ferroptosis through induction of oxidative injury [37]. In the present study, SIRT3 silencing significantly decreased LC3B-II accumulation and beclin1 protein expression, implicating SIRT3 as having a critical role as a positive regulator of autophagy. These observations were in direct contrast with a previous report that reduced SIRT3 expression enhanced autophagy flux [33]. Evidence supported the notion that excessive activation of autophagy promoted ferroptosis activator-induced induction of ferroptotic cell death $[16,23,24,36]$. Furthermore, a pharmacological approach or genetic inhibition of the autophagic pathway blocked ferroptotic cell death, 
at least in some instances. However, functional contributions of SIRT3 to ferroptosis have not been fully understood. We inferred that SIRT3-mediated autophagy, identified as an upstream mechanism, is required to regulate cellular iron homeostasis and cellular oxidative stress during ferroptosis, which was also apparent based on the aggravated effect of rapamycin and reversed effects of chloroquine, respectively.

It has been reported that SIRT3 can confer protection by regulating phosphorylation of AMPK, thereby inhibiting mTOR activity, which was paralleled by autophagy induction [38]. AMPK, with a critical role in lipophagy by initiation of chaperone-mediated autophagy and in governing cellular defenses against hypoxia, ischemia and oxidative stress [25, 39-41], serves as a regulator of cell survival or death. Given the well-documented positive role of AMPK activity in regulation of autophagy, we hypothesized that SIRT3 activates that AMPK-mTOR pathway and consequently induces autophagy. As expected, the present study clarified that increased SIRT3 expression upregulated the phosphorylation level of AMPK, whereas inhibited mTOR activity. AMPK is mechanistically implicated in the effect of SIRT3 on autophagy and the same effect was also confirmed in this study $[20,33,38]$. Compound $C$ was partially blocked the protein level of p-AMPK in SIRT3-induced circumstance, which was accompanied by decreases in LC3-II conversion as well as beclin1 protein level. Thus, it indicates that SIRT3 promotes induction of autophagy via activating the AMPK-mTOR pathway. Autophagy is activated, leading to lipid peroxidation and iron accumulation, in response to induction of ferroptosis [17, 22, 23]. Importantly, lysosomal dysfunction and uncontrolled or inappropriate autophagic flux are involved in iron dysbolism in ferroptosis $[16,23]$. The function of AMPK in mediation of ferroptosis is required for beclin1 phosphorylation, with inhibition of system $\mathrm{X}_{\mathrm{c}}{ }^{-}$activity being reported [25]. Perhaps AMPK represents a mechanistic link between ferroptosis and autophagy; this was supported by our data that pharmacological inhibition of AMPK partially abolished SIRT3-induced ferroptotic events in our in vitro model.

Emerging studies have revealed that downregulation of GPX4 could promote induction of ferroptosis, it depletion in cells and mice results in the accumulation of lipid peroxides and lipid ROS [42-44]. Our data suggest that inhibition of GPX4 induces ferroptosis by increasing lipid ROS level in trophoblastic cells. Moreover, we also found that depletion of SIRT3 blocked RSL3-induced ferroptosis, indicating that decreased SIRT3 prevented ferroptosis by increasing GPX4 level. In the present study, we elucidated a novel role of SIRT3 in autophagy and ferroptosis via regulating AMPK-mTOR pathway and GPX4 level. Taken together, our findings demonstrated a scenario where ferroptotic-inducing agents not only induced ferroptotic events, but also promoted autophagy activation, paralleled by increased SIRT3 expression. Thus, depletion of SIRT3 decreased the phosphorylation level of AMPK, whereas increased mTOR activity, eventually inhibiting autophagy activation as well as blocking induction of ferroptosis. Therefore, we inferred that mediation of SIRT3 on the p-AMPK-mTOR pathway partially contributed to regulated cell death, including autophagy and ferroptosis. In paraller, depletion of SIRT3 inhibited ferroptosis by increasing GPX4 level (Fig. 8). However, further investigations are needed to confirm whether the same mechanisms are responsible in vivo. Additionally, whether SIRT3 can regulate AMPK-mediated beclin1 
phosphorylation to promote ferroptosis by directly blocking system $\mathrm{X}_{\mathrm{c}}{ }^{-}$activity, and the crosstalk between AMPK and GPX4, also requires further study.

\section{Conclusions}

High glucose exposure of trophoblasts lead to upregulation of SIRT3 protein level, which in turn exacerbated ferroptotic-inducing compounds-triggered ferroptosis by positively regulating autophagy induction. We inferred that increased SIRT3 expression contributed to autophagy activation by activating AMPK-mTOR pathway, promoting iron accumulation and lipid peroxidation, and in turn, inducing ferroptosis. Whereas, decreased SIRT3 inhibits ferroptosis through regulating GPX4 level. Finally, SIRT3 deficiency was resistant to erastin-induced autophagy-dependent ferroptosis by inhibiting the AMPK/mTOR pathway and increasing GPX4 level in trophoblastic cells.

\section{Declarations}

\section{Ethics approval and consent to participate}

Not applicable

\section{Consent for publication}

Not applicable

\section{Availability of data materials}

The data that support the finding of this study are available from the corresponding author upon reasonable request.

\section{Competing interests}

There is no conflict of interest regarding the publication of this manuscript.

\section{Funding}

This work was financially supported by the National Key Research and Development Program of China (No. 2016YFD0500506), the Beijing Municipal Natural Science Foundation (No. S170001), the National Natural Science Foundation of China (No. 31630074, No. 31902170, No. 31972596), the China Postdoctoral Science Foundation (No. 2018M641547), the 111 Project (B16044), the Fundamental Research Funds for the Central Universities (No. 2019TC223, No. 2019TC120), and the Jinxinnong Animal Science Developmental Foundation.

\section{Authors' contributions}


DH and JW designed and conceived the research. DH, LJ, SH, JP, and YW performed the experiment. DH, XG and CF performed the analysis. DH wrote the manuscript. XG, JY and JW critically reviewed and revised the manuscript. All the authors read and approved the final manuscript.

\section{Acknowledgements}

We would like to thank Professor John P. Kastelic, University of Calgary, Calgary, AB, T2N 4N1, Canda, for his excellent suggestion about the writing and completion of the manuscript.

\section{Authors' information}

${ }^{1}$ State Key Laboratory of Animal Nutrition, College of Animal Science and Technology, China Agricultural University, Beijing 100193, P. R. China. ${ }^{2}$ College of Veterinary Medicine, Yunnan Agricultural University, Kunming 650201, P. R. China. ${ }^{3}$ Department of Obstetrics and Gynecology, China-Japan Friendship Hospital, Beijing 100029, P. R. China.

\section{Abbreviations}

AMPK: activated protein kinase; Baf-A1: bafilomycin A1; BSO: buthionine sulfoximine; CQ: chloroquine; GDM: gestational diabetes mellitus; GPX4: glutathione peroxidase 4; GSH: glutathione; MDA: malondialdehyde; mTORC1: rapamycin C1; ROS: reactive oxygen species; SIRT3: sirtuin 3; T2DM: type 2 diabetes mellitus.

\section{References}

1. Coustan DR. Gestational diabetes mellitus. Clin Chem. 2013;59:1310-21.

2. Murthi P, Vaillancourt C. Placental serotonin systems in pregnancy metabolic complications associated with maternal obesity and gestational diabetes mellitus. Biochim Biophys Acta Mol Basis Dis. 2019;7:

3. Dipla K, Triantafyllou A, Grigoriadou I, Kintiraki E, Triantafyllou GA, Poulios P, Vrabas IS, Zafeiridis A, Douma S, Goulis DG. Impairments in microvascular function and skeletal muscle oxygenation in women with gestational diabetes mellitus: links to cardiovascular disease risk factors. Diabetologia 2017;60:192-201.

4. Gauster M, Desoye G, Tötsch M, Hiden U. The placenta and gestational diabetes mellitus. Curr Diab Rep. 2012;12:16-23.

5. Schliefsteiner C, Peinhaupt M, Kopp S, Lögl J, Lang-Olip I, Hiden U, Heinemann A, Desoye G, Wadsack C. Human placental hofbauer cells maintain an anti-inflammatory M2 phenotype despite the presence of gestational diabetes mellitus. Front Immunol. 2017;8:

6. Chatuphonprasert W, Jarukamjorn K, Ellinger I. Physiology and pathophysiology of steroid biosynthesis, transport and metabolism in the human placenta. Front Pharmacol. 2018;9: 
7. Hübener C, Bidlingmaier M, Wu Z, Diebold J, Delius M, Friese K, Strasburger CJ, Hasbargen U. Human placental growth hormone: a potential new biomarker in gestational trophoblastic disease. Gynecol Oncol. 2015;136:264-8.

8. Subiabre M, Silva L, Toledo F, Paublo M, López MA, Boric MP, Sobrevia L. Insulin therapy and its consequences for the mother, foetus, and newborn in gestational diabetes mellitus. Biochim. Biophys Acta Mol Basis Dis. 2018;1864:2949-56.

9. Hernandez TL, Brand-Miller JC. Nutrition therapy in gestational diabetes mellitus: time to move forward. Diabetes Care 2018;41:1343-5.

10. Gao L, Qi HB, Kamana KC., Zhang XM, Zhang H, Baker PN. Excessive autophagy induces the failure of trophoblast invasion and vasculature: possible relevance to the pathogenesis of preeclampsia. $J$ Hypertens. 2015;33:106-17.

11. Yung HW, Alnæs-Katjavivi P, Jones CJ, El-Bacha T, Golic M, Staff AC, Burton GJ. Placental endoplasmic reticulum stress in gestational diabetes: the potential for therapeutic intervention with chemical chaperones and antioxidants. Diabetologia 2016;59:2240-50.

12. Peng HY, Li MQ, Li HP. High glucose suppresses the viability and proliferation of HTR-8/SVneo cells through regulation of the miR-137/PRKAA1/IL-6 axis. Int. J Mol Med. 2018;42:799-810.

13. Filomeni G, De Zio D, Cecconi F. Oxidative stress and autophagy: the clash between damage and metabolic needs. Cell Death Differ. 2015;22:377-88.

14. Zhuang T, Han H, Yang Z. Iron, oxidative stress and gestational diabetes. Nutrients 2014;6:3968-80.

15. Ji L, Chen Z, Xu Y, Xiong G, Liu R, Wu C, Hu H, Wang L. Systematic characterization of autophagy in gestational diabetes mellitus. Endocrinology. 2017;158:2522-32.

16. Tang D, Kang R, Berghe TV, Vandenabeele P, Kroemer G. The molecular machinery of regulated cell death. Cell Res. 2019;29:347-64.

17. Dixon SJ, Lemberg KM, Lamprecht MR, Skouta R, Zaitsev EM, Gleason CE, Patel DN, Bauer AJ, Cantley AM, Yang WS, et al. Ferroptosis: an iron-dependent form of nonapoptotic cell death. Cell 2012;149:1060-72.

18. Singh CK, Chhabra G, Ndiaye MA, Garcia-Peterson LM, Mack NJ, Ahmad N. The role of Sirtuins in antioxidant and redox signaling. Antioxid Redox Signal. 2018;28:643-61.

19. Bell EL, Guarente L. The Sirt3 divining rod points to oxidative stress. Mol Cell 2011;42:561-68.

20. Zhang M, Deng YN, Zhang JY, Liu J, Li YB, Su H, Qu QM. SIRT3 protects rotenone-induced injury in SH-SY5Y cells by promoting autophagy through the LKB1-AMPK-mTOR pathway. Aging Dis. 2018;9:273-86.

21. Novgorodov SA, Voltin JR, Gooz MA, Li L, Lemasters JJ, Gudz TI. Acid sphingomyelinase promotes mitochondrial dysfunction due to glutamate-induced regulated necrosis. J Lipid Res. 2018;59:31229.

22. Gao M, Monian P, Pan Q, Zhang W, Xiang J, Jiang X. Ferroptosis is an autophagic cell death process. Cell Res. 2016;26:1021-32. 
23. Wu Z, Geng Y, Lu X, Shi Y, Wu G, Zhang M, Shan B, Pan H, Yuan J. Chaperone-mediated autophagy is involved in the execution of ferroptosis. Proc Natl Acad Sci USA. 2019;116:2996-3005.

24. Hou W, Xie Y, Song X, Sun X, Lotze MT, Zeh HJ, Kang R, Tang D. Autophagy promotes ferroptosis by degradation of ferritin. Autophagy 2016;12:1425-28.

25. Song X, Zhu S, Chen P, Hou W, Wen Q, Liu J, Xie Y, Liu J, Klionsky DJ, Kroemer G, Lotze MT, Zeh HJ, Kang R, Tang D. AMPK-mediated BECN1 phosphorylation promotes ferroptosis by directly blocking system Xc- activity. Curr Biol. 2019;28:2388-99.

26. Lahiri V, Hawkins WD, Klionsky DJ. Watch what you (self-) eat: autophagic mechanisms that modulate metabolism. Cell Metab. 2019;29:803-26.

27. Stockwell BR, Friedmann Angeli JP, Bayir H, Bush Al, Conrad M, Dixon SJ, Fulda S, Gascón S, Hatzios SK, Kagan VE, et al. Ferroptosis: a regulated cell death nexus linking metabolism, redox biology, and disease. Cell 2017;171:273-85.

28. Feng H, Stockwell BR. Unsolved mysteries: How does lipid peroxidation cause ferroptosis? PLoS Biol. 2018;16:

29. Hirschhorn T, Stockwell BR. The development of the concept of ferroptosis. Free Radic Biol Med 2019;133:130-43.

30. Bai Y, Meng L, Han L, Jia Y, Zhao Y, Gao H; Kang R, Wang X, Tang D, Dai E. Lipid storage and lipophagy regulates ferroptosis. Biochem Biophys Res Commun. 2019;508:997-1003.

31. Liu J, Li D, Zhang T, Tong Q, Ye RD, Lin L. SIRT3 protects hepatocytes from oxidative injury by enhancing ROS scavenging and mitochondrial integrity. Cell Death Dis. 2017;8:

32. Xu X, Zhu XP, Bai JY, Xia P, Li Y, Lu Y, Li XY, Gao X. Berberine alleviates nonalcoholic fatty liver induced by a high-fat diet in mice by activating SIRT3. FASEB J. 2019;33:7289-300.

33. Li S, Dou X, Ning H, Song Q, Wei W, Zhang X, Shen C, Li J, Sun C, Song Z. Sirtuin 3 acts as a negative regulator of autophagy dictating hepatocyte susceptibility to lipotoxicity. Hepatology 2017;66:93652.

34. Dikalova AE, Itani HA, Nazarewicz RR, McMaster WG, Flynn CR, Uzhachenko R, Fessel JP, Gamboa JL, Harrison DG, Dikalov SI. Sirt3 impairment and SOD2 hyperacetylation in vascular oxidative stress and hypertension. Circ. Res. 2017;121:564-74.

35. Kang R, Tang D. Autophagy and ferroptosis - what's the connection? Curr Pathobiol Rep. 2017;5:15359.

36. Zhou B, Liu J, Kang R, Klionsky DJ., Kroemer G, Tang D. Ferroptosis is a type of autophagydependent cell death. Semin Cancer Biol. 2019; https://doi.org/10.1016/j.semcancer.2019.03.002.

37. Yang M, Chen P, Liu J, Zhu S, Kroemer G, Klionsky DJ, Lotze MT, Zeh HJ, Kang R, Tang D. Clockophagy is a novel selective autophagy process favoring ferroptosis. Sci Adv. 2019;5:

38. Dai SH, Chen T, Li X, Yue KY, Luo P, Yang LK, Zhu J, Wang YH, Fei Z, Jiang XF. Sirt3 confers protection against neuronal ischemia by inducing autophagy: Involvement of the AMPK-mTOR pathway. Free Radic Biol Med. 2017;108:345-53. 
39. Qi D, Young LH. AMPK: energy sensor and survival mechanism in the ischemic heart. Trends Endocrinol Metab. 2015;26:422-9.

40. Moral-Sanz J, Lewis SA, MacMillan S, Ross FA, Thomson A, Viollet B, Foretz M, Moran C, Hardie DG, Evans AM. The LKB1-AMPK- $a 1$ signaling pathway triggers hypoxic pulmonary vasoconstriction downstream of mitochondria. Sci. Signal. 2019;11: https://doi.org/10.1126/scisignal.aau0296.

41. Kaushik S, Cuervo AM. AMPK-dependent phosphorylation of lipid droplet protein PLIN2 triggers its degradation by CMA. Autophagy 2016;12:432-8.

42. Bersuker K, Hendricks J, Li Z, Magtanong L, Ford B, Tang PH, Roberts MA, Tong B, Maimone TJ, Zoncu R, et al. The CoQ oxidoreductase FSP1 acts parallel to GPX4 to inhibit ferroptosis. Nature. 2019;575:688-92.

43. Park TJ, Park JH, Lee GS, Lee JY, Shin JH, Kim MW, Kim YS, Kim JY, Oh KJ, Han BS, et al. Quantitative proteomic analyses reveal that GPX4 downregulation during myocardial infarction contributes to ferroptosis in cardiomyocytes. Cell Death Dis. 2019;10:835.

44. Maiorino M, Conrad M, Ursini F. GPx4, lipid peroxidation, and cell death: discoveries, rediscoveries, and open Issues. Antioxid Redox Signal. 2018;29:61-74.

\section{Figures}

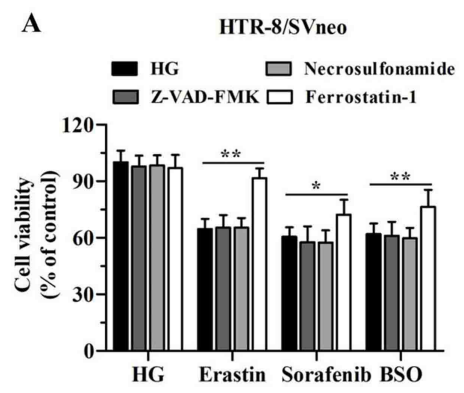

C

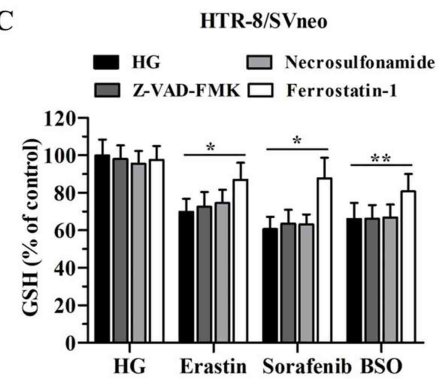

E

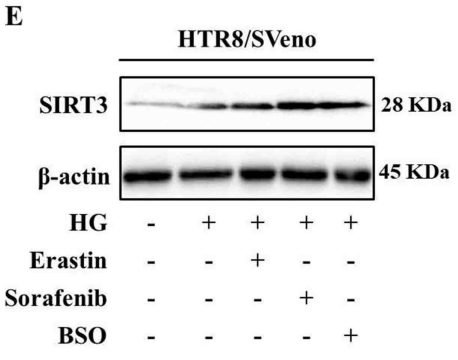

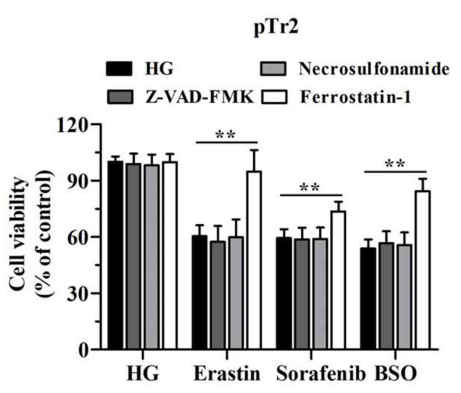

pTr2
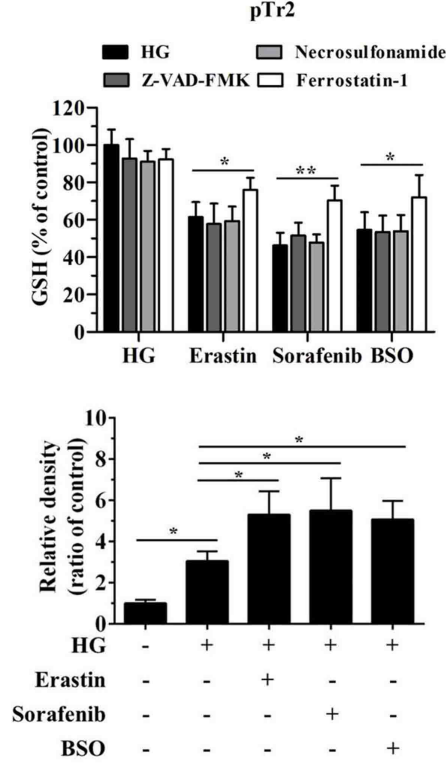

B

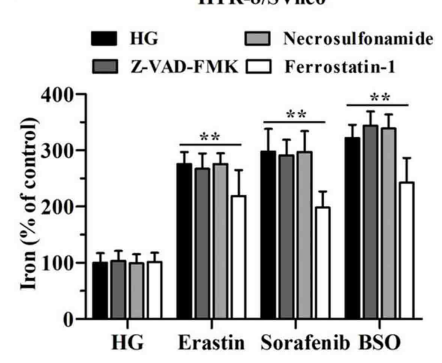

D
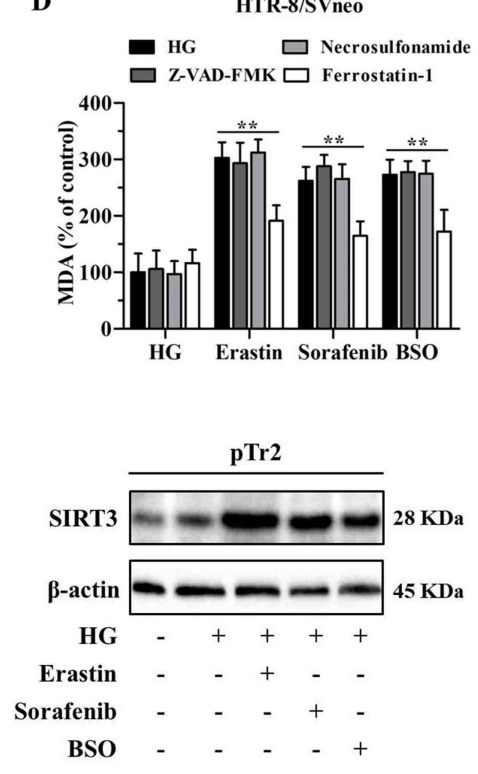

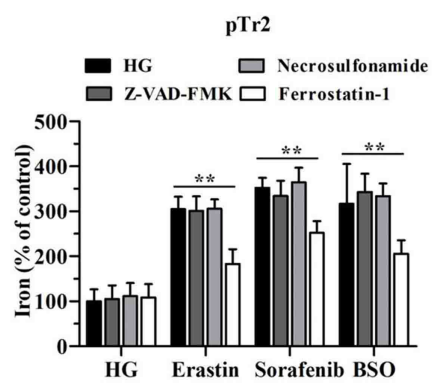

pTr2
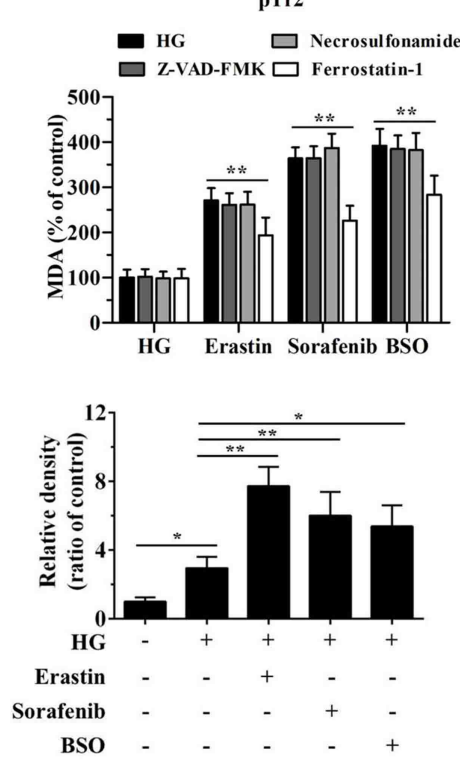

Figure 2 
SIRT3 is increased during trophoblast ferroptosis. HTR8/SVeno and pTr2 cells were treated with erastin $(20 \mu \mathrm{M}$ for HTR8/SVeno cells; $10 \mu \mathrm{M}$ for pTr2 cells), sorafenib $(10 \mu \mathrm{M})$ and BSO (200 $\mu \mathrm{M}$ for

HTR8/SVeno cells; $100 \mu \mathrm{M}$ for pTr2 cells) with or without indicated inhibitors (ZVAD-FMK, $10 \mu \mathrm{M}$;

necrosulfonamide, $1 \mu \mathrm{M}$; and ferrostatin-1, $2 \mu \mathrm{M}$ ) for $24 \mathrm{~h}$ in the presence of high glucose. a Cell viability was determined by CCK8 assay. b Iron concentration was measured with an Iron Assay kit. c GSH content in cell lysates was assayed by Total Glutathione Quantification Kit. d MDA was detected with colorimatria MDA Assay Kit. HTR8/SVeno and pTr2 cells were treated with erastin $(20 \mu \mathrm{M}$ for HTR8/SVeno cells; 10 $\mu \mathrm{M}$ for $\mathrm{pTr} 2$ cells), sorafenib $(10 \mu \mathrm{M})$, and BSO (200 $\mu \mathrm{M}$ for HTR8/SVeno cells; $100 \mu \mathrm{M}$ for pTr2 cells) for $24 \mathrm{~h}$ in the presence or absence of high glucose. e SIRT3 protein expression was determined by western blot analysis. GSH: glutathione, MDA: malondialdehyde, HG: high glucose, BSO: buthionine sulfoximine. Data are presented as means $\pm \mathrm{SD}$. ${ }^{*} \mathrm{P}<0.05 ; * * \mathrm{P}<0.01$

A
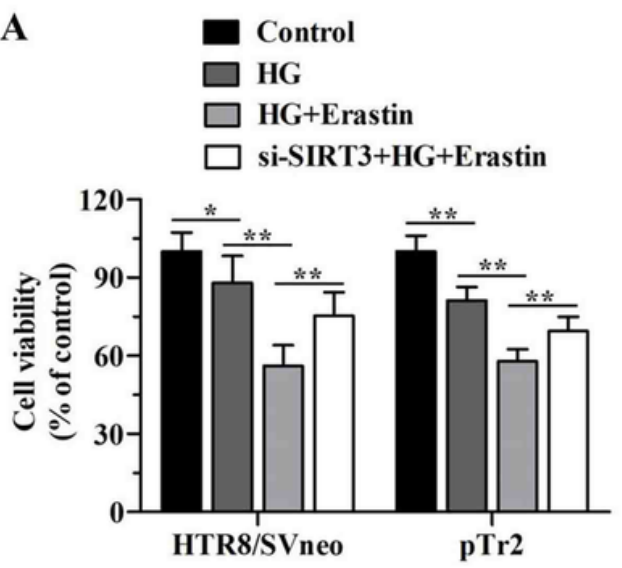

D
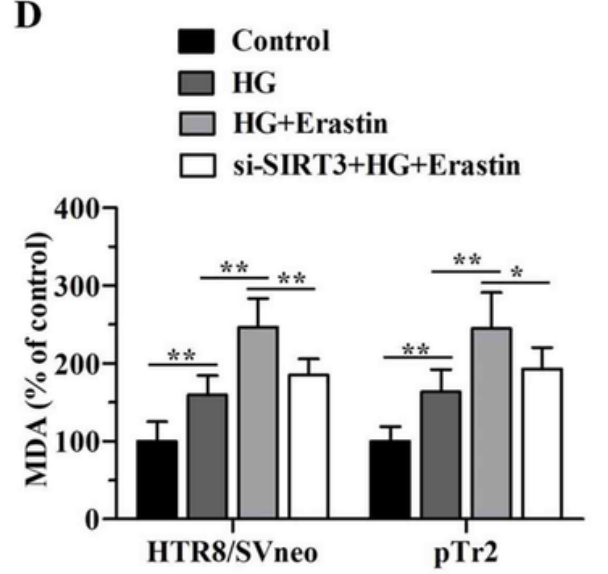

B
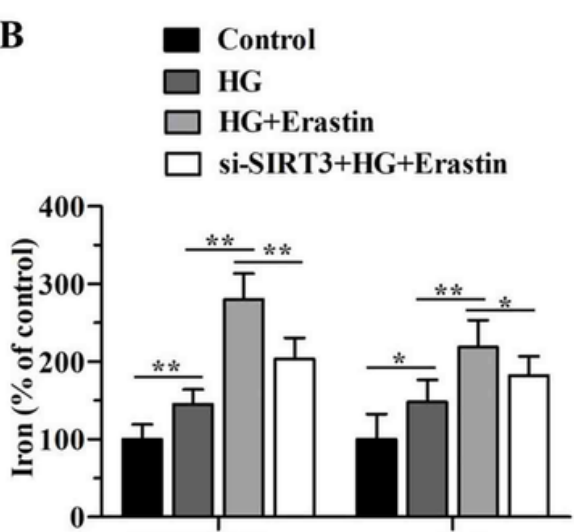

HTR8/SVneo

$\mathbf{E}$
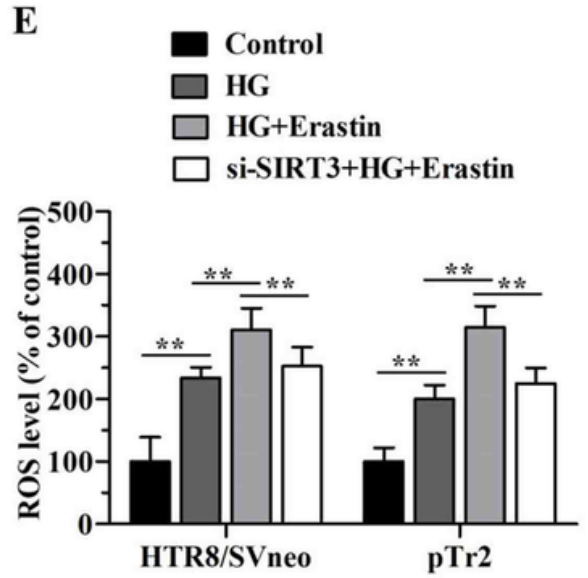
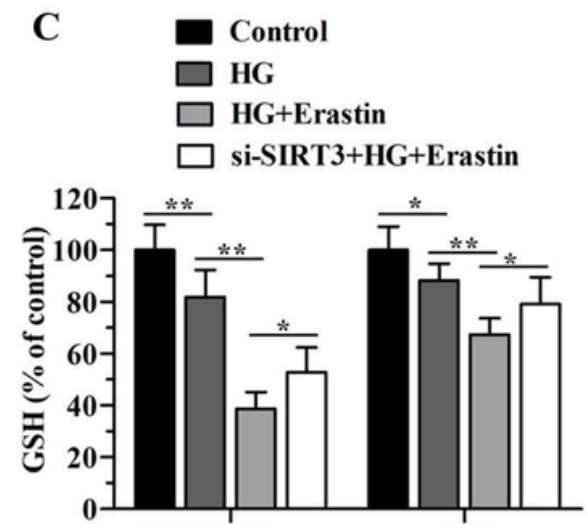

HTR8/SVneo

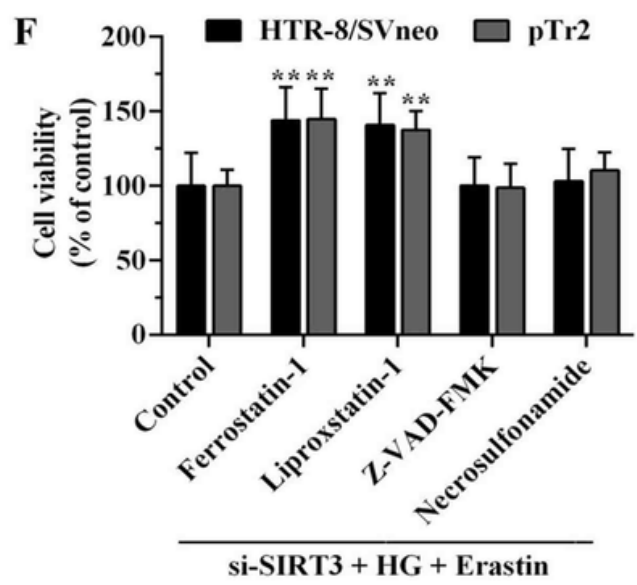

Figure 4

Increased SIRT3 expression contributes to trophoblast ferroptosis. HTR8/SVeno and pTr2 cells were transfected with control siRNA or SIRT3 siRNA. Subsequently, transfected cells were treated with erastin $(20 \mu \mathrm{M}$ for HTR8/SVeno cells; $10 \mu \mathrm{M}$ for pTr2 cells) for $24 \mathrm{~h}$ in the presence of high glucose. a Cell viability was determined by CCK8 assay. b Iron concentration was measured by Iron Assay kit. c GSH content in cell lysates was assayed by Total Glutathione Quantification Kit. d MDA was detected by colorimatria MDA Assay Kit. e Intracellular ROS generation was detected with DCFH-DA probe. The indicated SIRT3 knockdown trophpblastic cells were treated with erastin $(20 \mu \mathrm{M}$ for HTR8/SVeno cells; 10 
$\mu \mathrm{M}$ for pTr2 cells), with or without the indicated inhibitors (ZVAD-FMK, $10 \mu \mathrm{M}$; necrosulfonamide, $1 \mu \mathrm{M}$; ferrostatin-1, $2 \mu \mathrm{M}$ ) for $24 \mathrm{~h}$ in the presence of high glucose. $\mathrm{f}$ Cell viability was determined by CCK8 assay. Data are presented as means $\pm \mathrm{SD}$. ${ }^{*} \mathrm{P}<0.05 ; * * \mathrm{P}<0.01$.

A

HTR8/SVeno

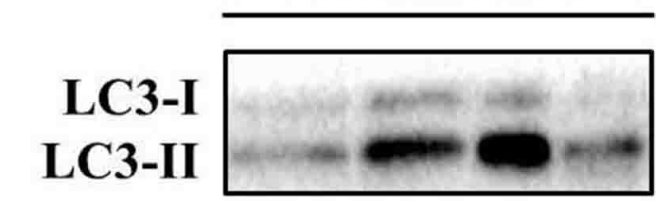

Beclin 1
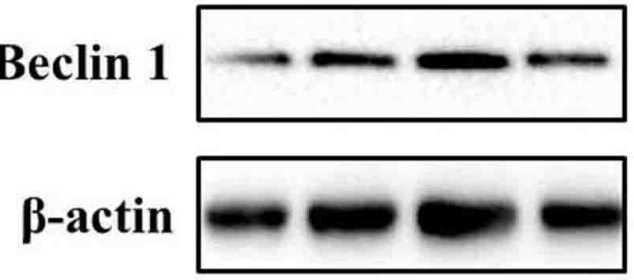

HG - $\quad+\quad++$

Erastin - $\quad+\quad+$ si-SIRT3 - $3 \quad-\quad+$

B

\section{pTr2}

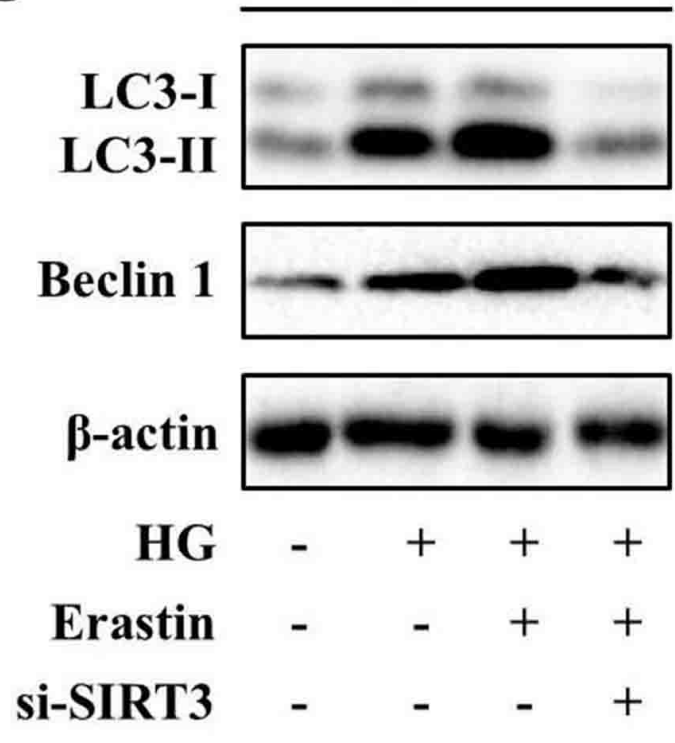

$16 \mathrm{KDa}$ 14 KDa

51 KDa

$45 \mathrm{KDa}$

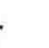

$16 \mathrm{KDa}$ 14 KDa 45 KDa

\section{Figure 5}

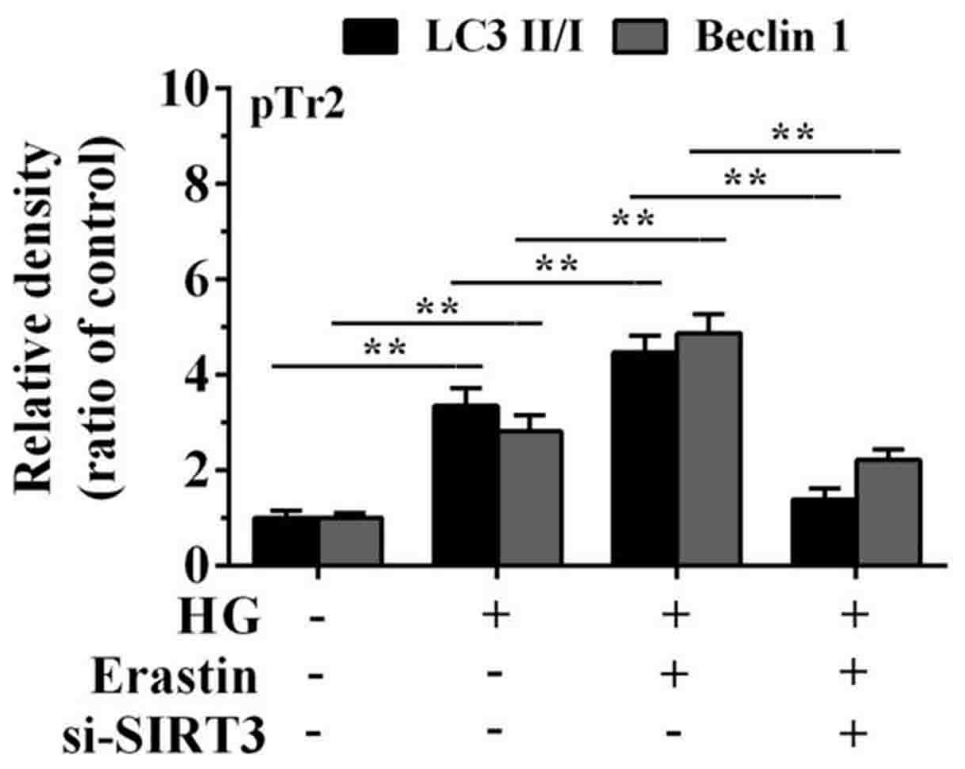

Increased SIRT3 expression promotes autophagy activation. HTR8/SVeno and pTr2 cells were transfected with control siRNA or SIRT3 siRNA. Subsequently, transfected cells were treated with erastin ( $20 \mu \mathrm{M}$ for HTR8/SVeno cells; $10 \mu \mathrm{M}$ for pTr2 cells) for $24 \mathrm{~h}$ in the presence of high glucose. Protein levels of LC3-I/II (a) and beclin1 (b) were determined by western blot analysis. Data are presented as means $\pm S D$. ${ }^{*} P<0.05 ; * \star P<0.01$ 

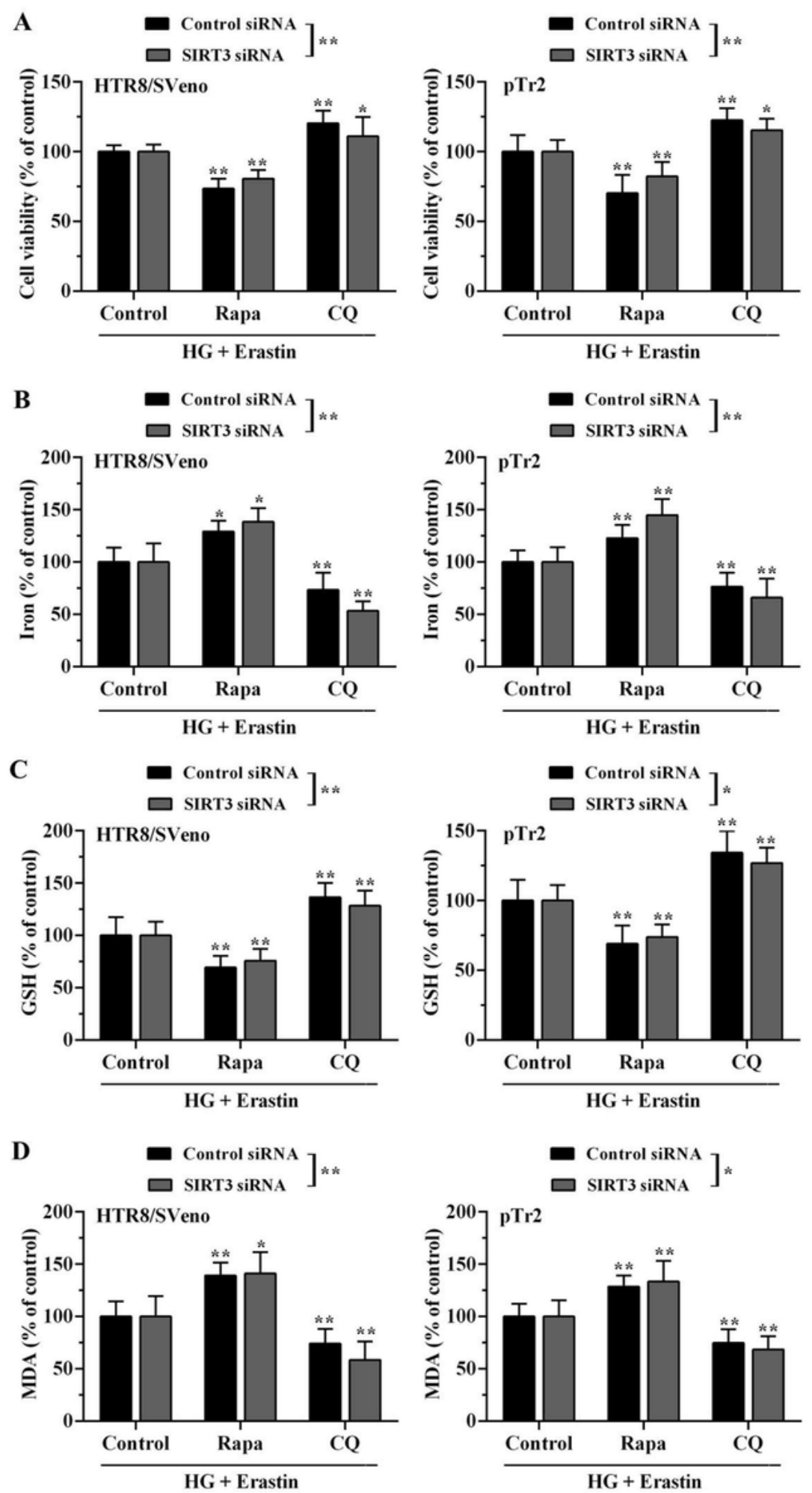

\section{Figure 7}

Enhanced ferroptosis by SIRT3 is associated with autophagy activation. HTR8/SVeno and pTr2 cells were transfected with control siRNA or SIRT3 siRNA. Subsequently, transfected cells were treated with erastin ( $20 \mu \mathrm{M}$ for HTR8/SVeno cells; $10 \mu \mathrm{M}$ for pTr2 cells), with or without the rapamycin $(500 \mathrm{nM})$, chloroquine $(200 \mathrm{nM})$, and bafilomycin $(50 \mathrm{nM})$ for $24 \mathrm{~h}$ in the presence of high glucose. a Cell viability was determined by CCK8 assay. b Iron concentration was measured by Iron Assay kit. c GSH content in 
cell lysates was assayed by Total Glutathione Quantification Kit. d MDA level was detedted by colorimatria MDA Assay Kit. Rapa: rapamycin, CQ: chloroquine. Data are presented as means $\pm \mathrm{SD}$. ${ }^{*}, \mathrm{P}<$ 0.05 ; **, $\mathrm{P}<0.01$.

A

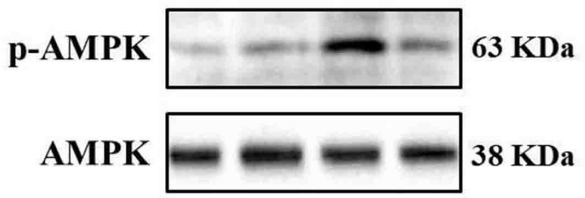

HG -+++

Erastin - - + +

Si-SIRT3 - - - +

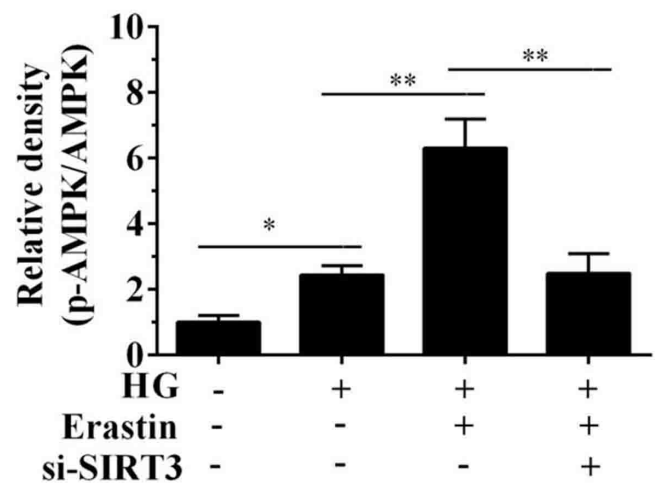

C

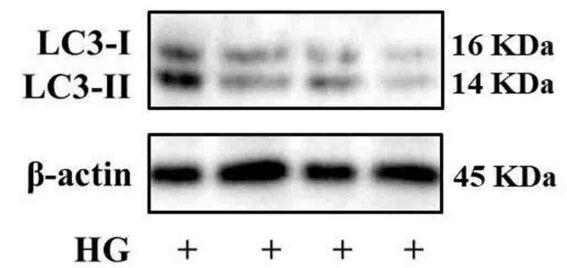

Erastin ++++

Compound C $\frac{-\quad+}{\text { si-Control si-SIRT3 }}$

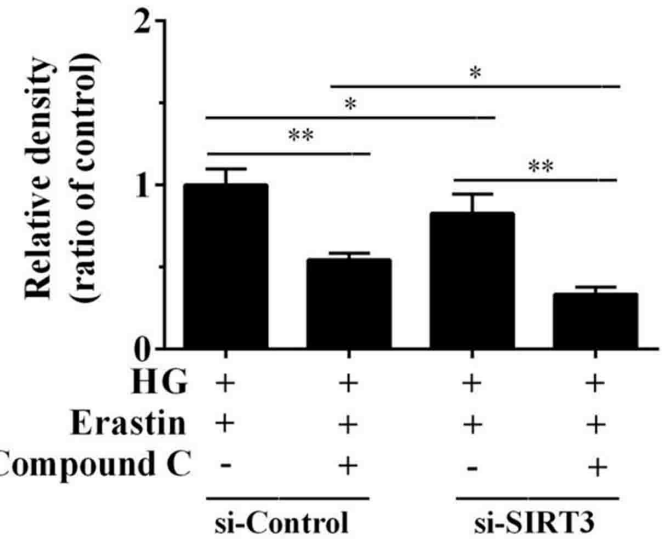

B

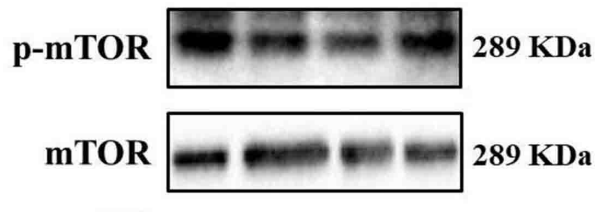

HG $\quad-\quad+\quad+\quad+$

Erastin - - ++

si-SIRT3 - 3 - +

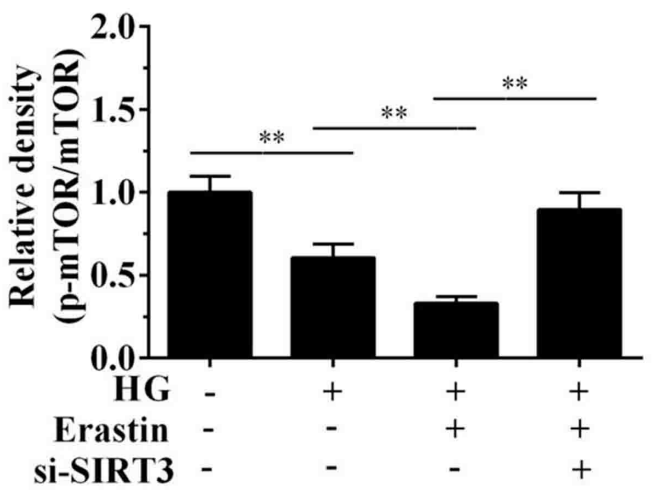

D

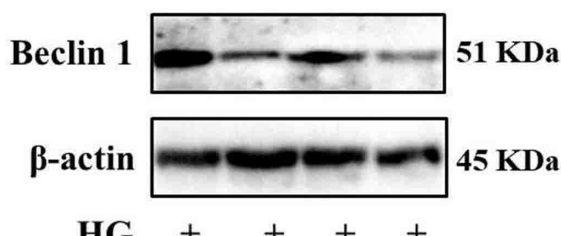

Erastin ++++

Compound C -++

si-Control si-SIRT3

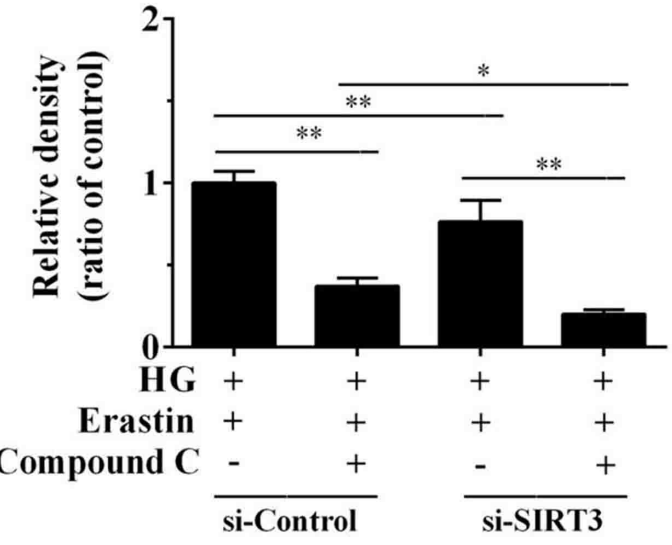

Figure 10

SIRT3 upregulates autophagy by promoting AMPK. HTR8/SVeno cells were transfected with control siRNA or SIRT3 siRNA. Subsequently, transfected cells were treated with erastin $(20 \mu \mathrm{M})$ for $24 \mathrm{~h}$ in the 
presence of high glucose. $(a, b)$ Protein levels of AMPK, p-AMPK, mTOR and p-mTORwere determined by western blot analysis. The indicated SIRT3 knockdown trophpblastic cells were treated with erastin (20 $\mu \mathrm{M})$ with or without compound $\mathrm{C}(5 \mu \mathrm{M})$ for $24 \mathrm{~h}$ in the presence of high glucose. (c,d) Protein levels of LC3-I/II and beclin1 were determined by western blot analysis. Data are presented as means \pm SD. $* P<$ $0.05 ; * * \mathrm{P}<0.01$

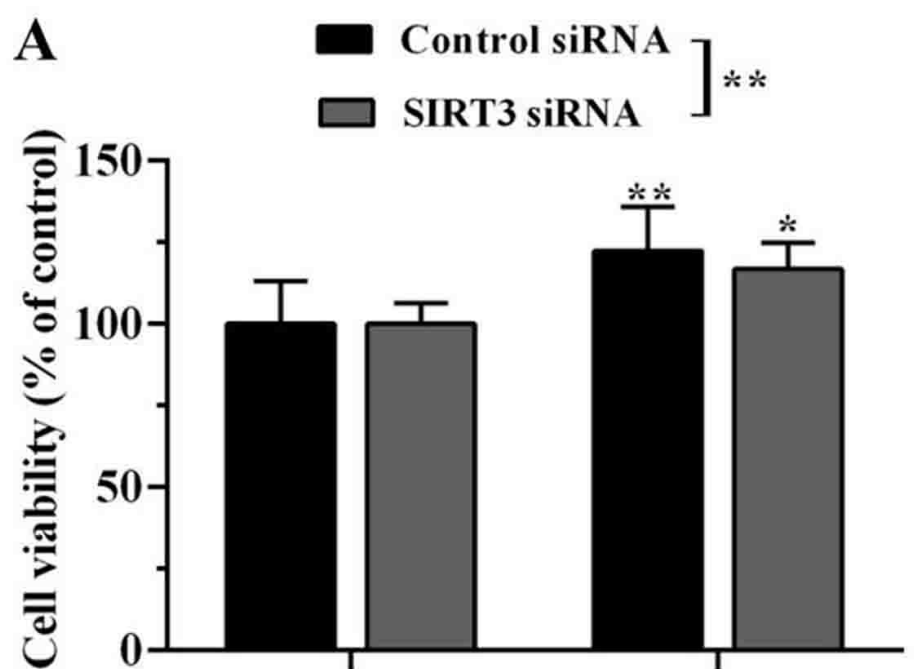

Control

Compound C

HG + Erastin

C
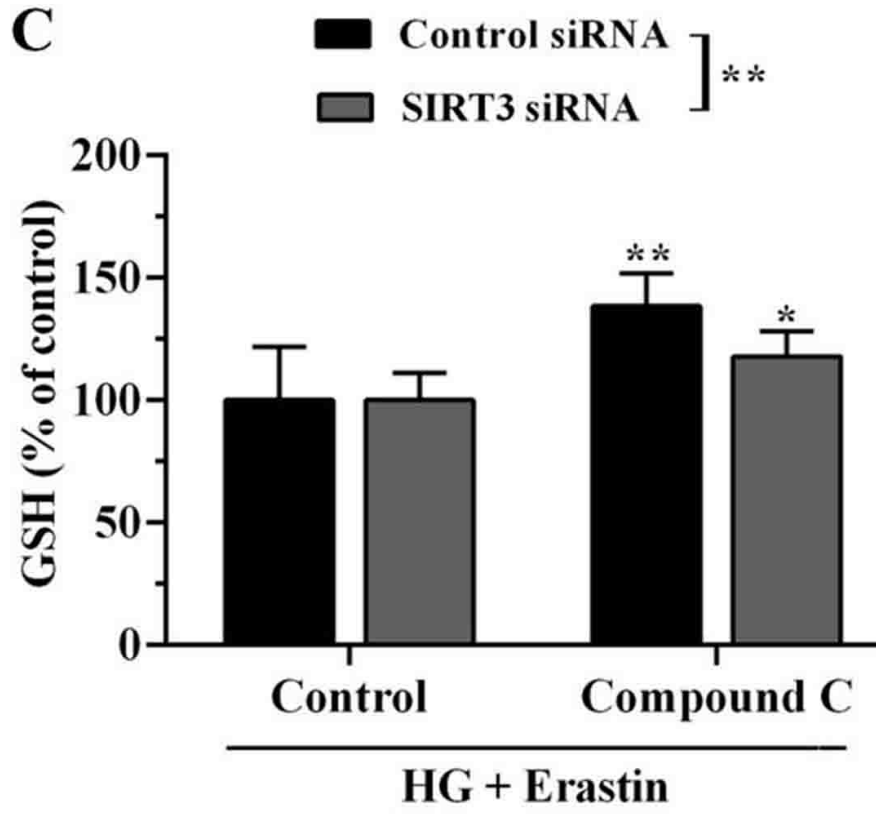
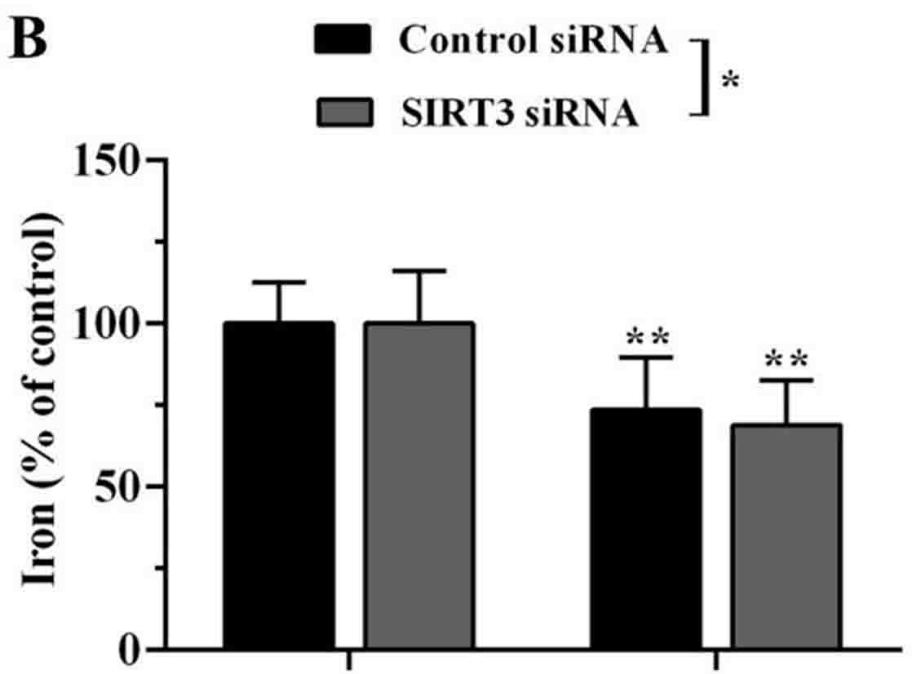

Control

Compound C

HG + Erastin
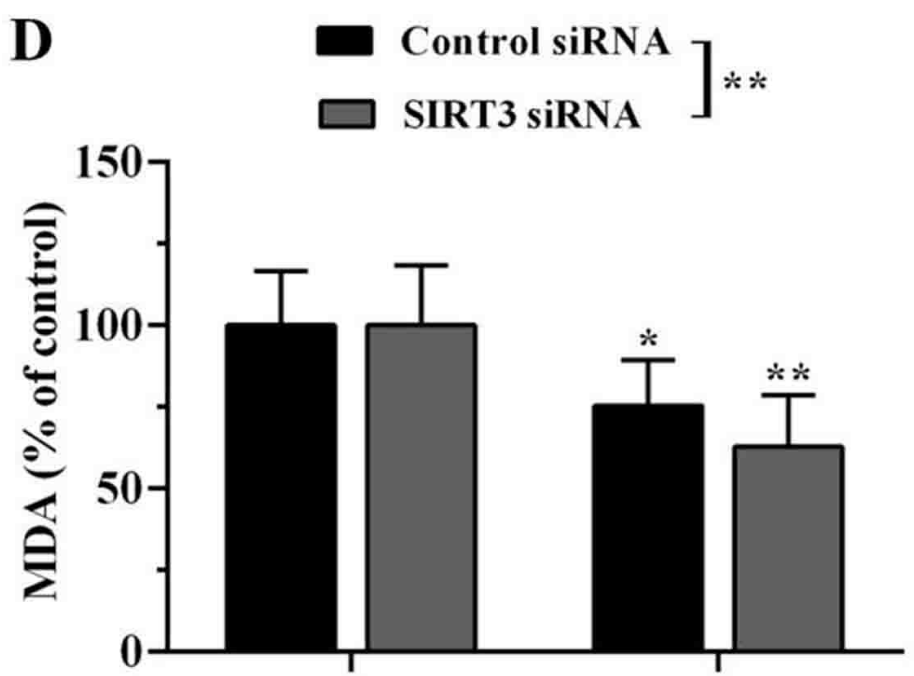

Control
Compound C

HG + Erastin

Figure 12

Suppression of AMPK impairs SIRT3-enhanced ferroptpsis. HTR8/SVeno cells were transfected with control siRNA or SIRT3 siRNA. Subsequently, transfected cells were treated with erastin $(20 \mu \mathrm{M})$ with or without compound $\mathrm{C}(5 \mu \mathrm{M})$ for $24 \mathrm{~h}$ in the presence of high glucose. a Cell viability was determined by CCK8 assay. b Iron concentration was measured by Iron Assay kit. c GSH content in cell lysates was 


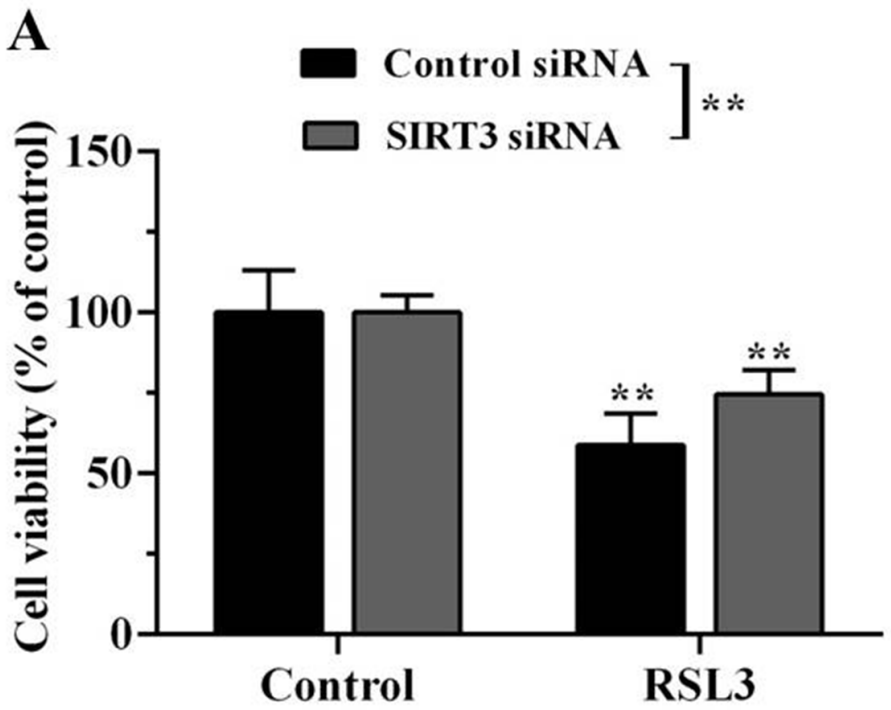

HG + Erastin

C
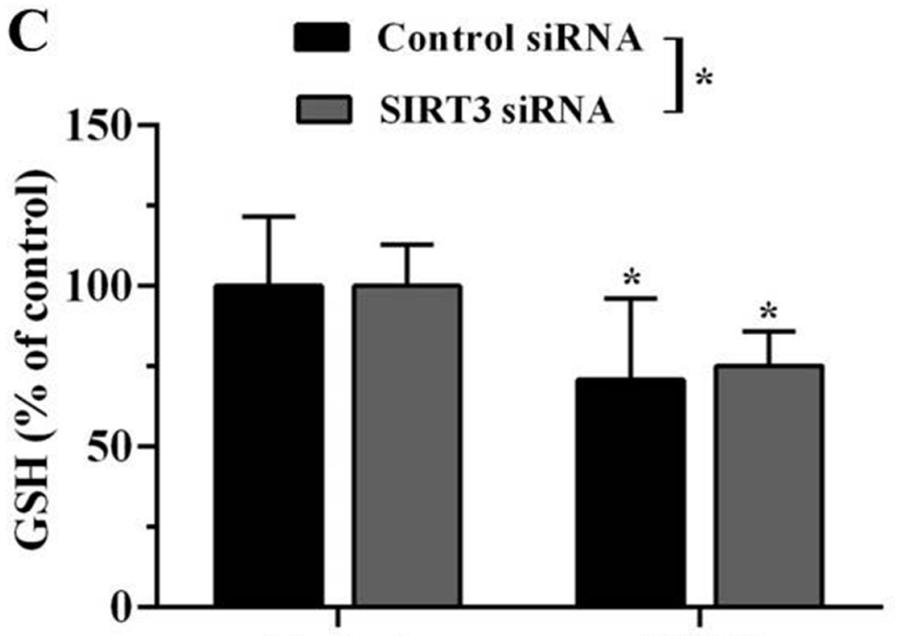

Control
B

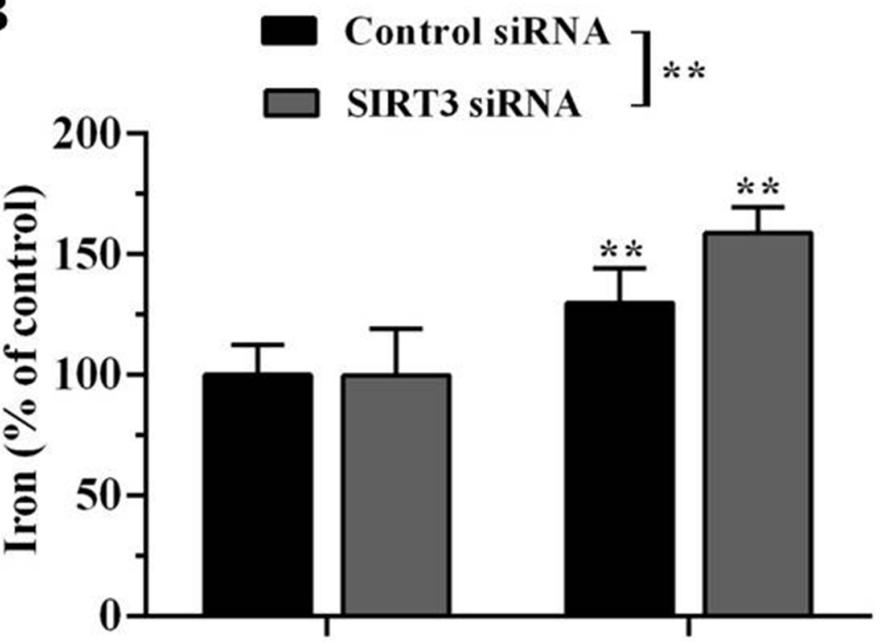

Control

HG + Erastin

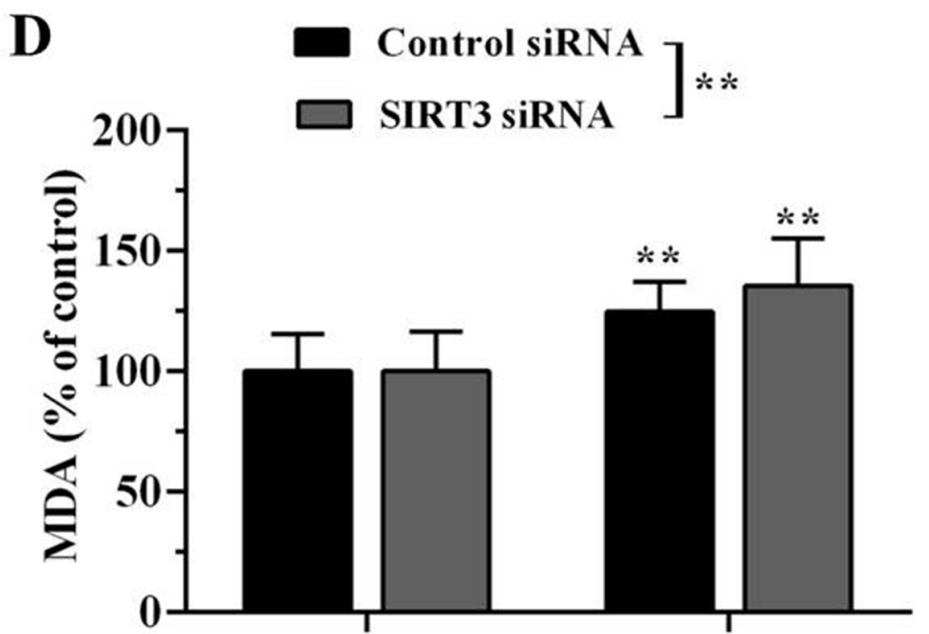

Control

RSL3

HG + Erastin

HG + Erastin

Figure 13

Depletion of SIRT3 inhibits ferroptosis by increasing GPX4 level. HTR8/SVeno cells were transfected with control siRNA or SIRT3 siRNA. Subsequently, transfected cells were treated with erastin $(20 \mu \mathrm{M})$ with or without RSL3 $(3 \mu \mathrm{M})$ for $24 \mathrm{~h}$ in the presence of high glucose. a Cell viability was determined by CCK8 assay. b Iron concentration was measured by Iron Assay kit. c GSH content in cell lysates was assayed by Total Glutathione Quantification Kit. d MDA level was detected by colorimatria MDA Assay Kit. Data are presented as means \pm SD. ${ }^{*} \mathrm{P}<0.05 ; * \star \mathrm{P}<0.01$ 


\section{High glucose Erastin}

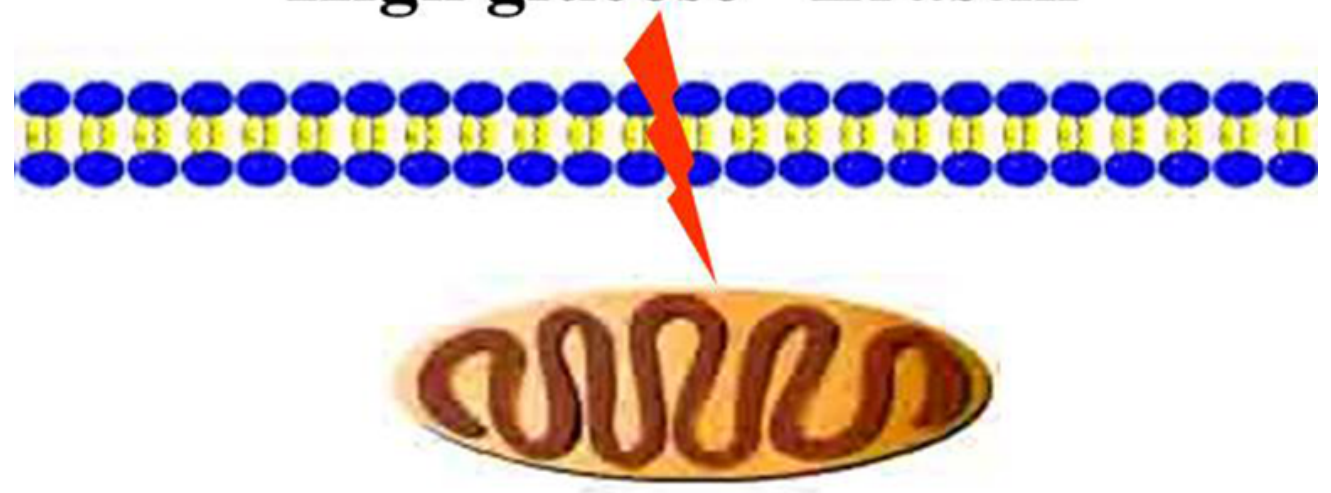

\section{Mitochondral dysfunction}

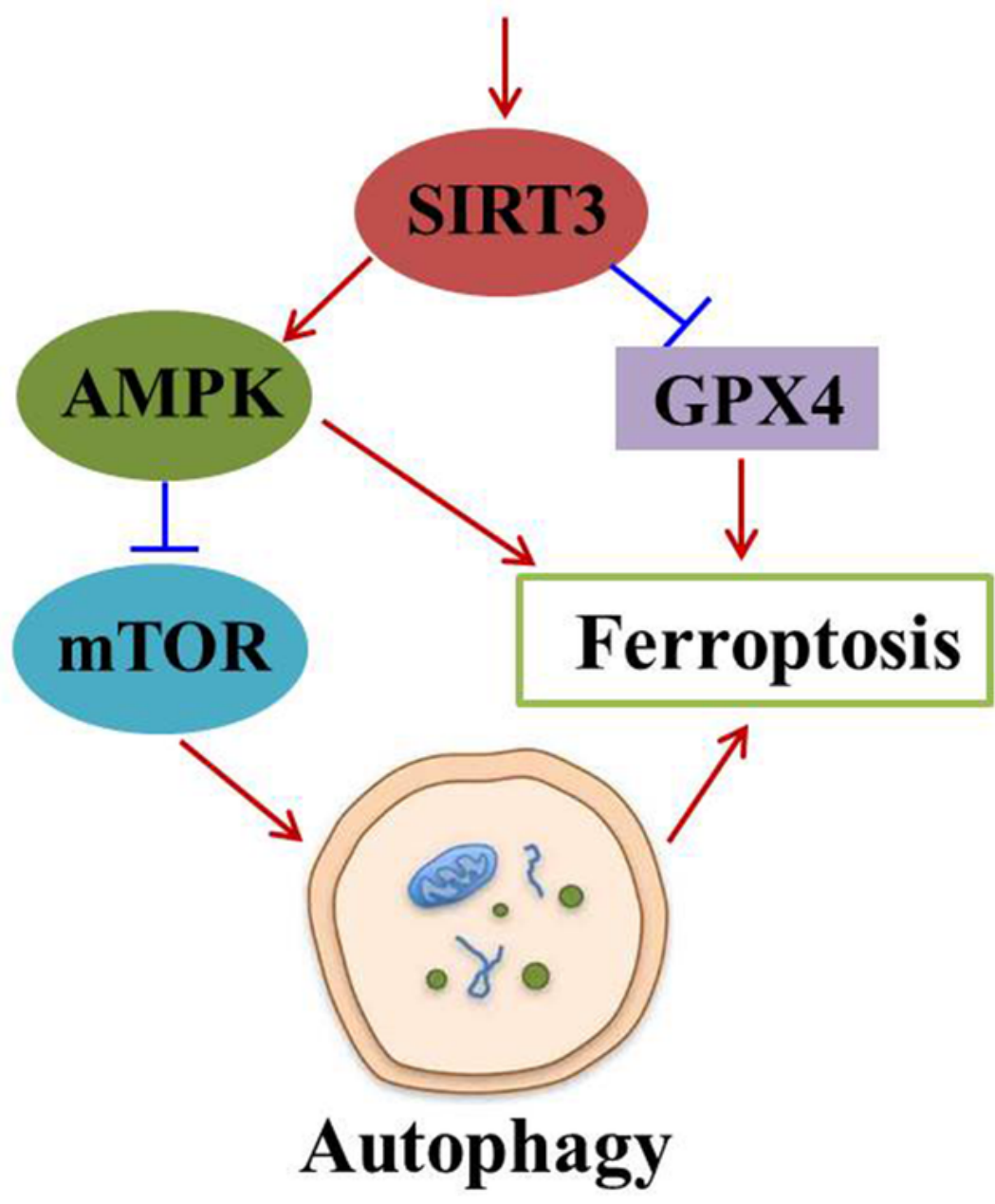

Figure 16

A proposed signaling pathway of SIRT3 involves in autophagy-dependent ferroptosis. Increased SIRT3 expression during mitochondrial dysfunction promotes autophagy through activating AMPK-mTOR pathway and eventually leads to ferroptosis in trophoblastic cells. Red arrow indicates activation whereas blue symbol indicates inhibition. 


\section{Supplementary Files}

This is a list of supplementary files associated with this preprint. Click to download.

- FigureS2.tif

- FigureS1.tif

- FigureS4.tif

- FigureS3.tif

- Figures2.tif

- FigureS1.tif

- Figures4.tif

- SupplementaryMaterial.docx

- FigureS3.tif

- FigureS5.tif

- FigureS5.tif

- SupplementaryMaterial.docx 